\title{
Lettre à la rédaction : Approche médico-légale d'un contrôle urinaire positif à la nandrolone
}

La nandrolone ou 19-nortestostérone est un dérivé synthétique de la testostérone. Elle permet une augmentation du poids du corps, de la masse musculaire et une augmentation de la force. Elle doit, pour cela, être accompagnée d'un entraînement approprié et d'un régime alimentaire riche en protéines. Cette substance augmente également l'agressivité et la confiance en soi, mais conduit à long terme à de l'instabilité émotionnelle. La nandrolone agit également comme cicatrisant, ce qui permet une récupération physique plus rapide en réparant les microtraumatismes de l'entraînement et améliore la densité osseuse. Enfin, elle diminue les douleurs articulaires.

La seule spécialité pharmaceutique contenant de la nandrolone encore inscrite à la Pharmacopée française est un collyre, le Kératyl. Le Trophobolène ( $1^{\text {er }}$ juillet 1997) et le Dynabolon ( $1^{\text {er }}$ novembre 1997) ont été retirés du marché.

L'administration orale d'un sel de nandrolone (sulfate) ou intramusculaire d'une forme estérifiée (décanoate ou undécanoate) conduit à la formation de 2 métabolites urinaires majeurs, la norandrosterone (NA) et la noretiocholanolone (NE), dont la durée de détection varie selon la voie d'administration (1). Après administration orale, la NA et la NE sont détectables 4-5 jours, tandis qu'après administration unique IM de $50 \mathrm{mg}$ de nandrolone undécanoate, ces métabolites sont encore retrouvés 8 mois après. On observe toujours une prédominance de la NA sur la NE.

La nandrolone est inscrite sur la liste des substances interdites (classe $\mathrm{C}$ ) du code antidopage du mouvement olympique. Est qualifiée de positive pour la nandrolone ou pour un autre 19-norstéroïde, une urine où il aura été mis en évidence la présence de NA, dont le signal chromatographique sera plus important que celui diun calibrateur à $2 \mathrm{ng} / \mathrm{ml}$ pour les hommes ou à $5 \mathrm{ng} / \mathrm{ml}$ pour les femmes. Le dosage urinaire se fait par chromatographie en phase gazeuse couplée à la spectrométrie de masse et ne pose pas de difficultés particulières.

En utilisant 3 techniques d'analyse totalement diffé- 
rentes sur une population d'environ 130 hommes, plusieurs auteurs (1-3) ont clairement établi qu'il existait une sécrétion naturelle de NA, dont les concentrations n'excédait pas $0,6 \mathrm{ng} / \mathrm{ml}$, soit une valeur largement inférieure au "seuil" de $2 \mathrm{ng} / \mathrm{ml}$. Plus récemment, Galan Martin et al (4) ont observé des concentrations flirtant avec les $20 \mathrm{ng} / \mathrm{ml}$, mais dans des populations difficiles à contrôler.

Dès 1997, la Food and Drug Administration (FDA) américaine autorisait la mise sur le marché d'anabolisants, en temps que supplément alimentaires pour augmenter les performances physiques. Ces substances sont proposées à la vente sans restriction. Elles sont interdites en France, mais peuvent aisément se commander par l'Internet. Deux composés sont classés parmi les 19-norstérö̈des, soit la 19-norandrostendione et le 19-norandrostendiol et sont inscrits sur la liste des substances interdites du CIO. Leur administration orale, généralement sous forme de gélule dosée à 50 ou $100 \mathrm{mg}$, conduit à la formation de NA et de NE $(1,5)$. Ces 2 métabolites sont détectables pendant 7 à 10 jours, avec des concentrations initiales très importantes, supérieures à $10000 \mathrm{ng} / \mathrm{ml}$. Ce type de concentrations n'est jamais observé après injection IM d'une préparation de nandrolone. En début d'élimination, la NA domine la NE, mais ce rapport va s'inverser plusieurs fois dans le temps. Une simple analyse urinaire ne permet donc pas d'affirmer sans ambiguïté un usage de supplément. En regard du code médical du CIO, l'origine précise de l'agent (nandrolone ou supplément) ayant conduit à la formation de NA est sans importance puisque tous ces produits sont interdits.

Sur le plan médico-légal, il peut être utile de déterminer finement l'agent dopant. Dans ces conditions, seule l'analyse d'une mèche de cheveux permettra cette discrimination, du fait de l'incorporation préférentielle de

\section{Références}

1. Kintz P., Cirimele V., Ludes B., Norandrosterone et noretiocholanolone : les métabolites révélateurs. Acta Clinica Belgica 1999 ; suppl. 1 : 68-73.

2. Le Bizec B., Monteau F., Gaudin I., André F., Evidence for the presence of endogenous 19-norandrosterone in human urine. J. Chromatogr. B $1999 ; 723$ : 157-72.

3. Dehennin L., Bonnaire Y., Plou P., Urinary excretion of 19-norandrosterone of endogenous origin in man : quantitative analysis by gas chromatography-mass spectrometry. J. Chromatogr. B $1999 ; 721: 301-7$.

4. Galan Martin A.M., Maynar Marino J.I., Garcia de Tiedra M.P. et al, Determination of nandrolone and metabolites in urine samples from sedentary persons and sportsmen. J. Chromatogr. B 2001 ; 761 : 229-36. la substance mère et non pas de ses métabolites dans la matrice pilaire(6).

L'analyse urinaire dans le cas d'une exposition à un 19-norstéroïde mettra en évidence la NA, sans que l'on puisse attribuer l'origine de ce métabolite à une substance particulière. Au contraire, on retrouvera dans les cheveux la nandrolone, ses esters ou la norandrostendione ou la norandrostendiol après une consommation répétée, ce qui permettra de caractériser de façon indiscutable l'agent dopant.

La nandrolone a pu être identifiée dans les cheveux humains (6-9), mais les concentrations généralement mesurées sont faibles, de l'ordre de quelques $\mathrm{pg} / \mathrm{mg}$, ce qui nécessite des procédures très sensibles.

A ce jour, l'analyse des cheveux n'est pas reconnue par le CIO, mais autorisée par le décret $n^{\circ}$ 2001-35 du 11 janvier 2001 relatif aux examens et prélèvements autorisés pour la lutte contre le dopage.

L'approche médico-légale d'un dossier visant à documenter un contrôle antidopage positif est donnée par l'arbre décisionnel suivant :

1 - Analyse des urines après le contrôle (3-6 semaines) - analyse positive : usage d'un ester de la nandrolone (du fait d'une élimination très lente)

- analyse négative : seule la voie orale peut être envisagée : 19-norstéroides, soit volontairement, soit par contamination de la nourriture (viande) ou des suppléments de type vitamines, acides aminés ...

2 - Analyse des cheveux

- analyse positive : usage répété d'un 19-norstéroide, avec discrimination du produit

- analyse négative : usage unique d'un 19-norstéroide (ne positivant pas les cheveux) ou contamination ponctuelle alimentaire ou par les suppléments (cas les plus fréquents).

5. Uralets V.P., Gillette P.A., Over-the-counter anabolic steroids 4-androsten-3,17-dione ; 4-androsten-3ß,17ß-diol; and 19-nor-4-androsten-3,17-dione : excretion studies in men. J. Anal. Toxicol. $1999 ; 23$ : 357-66.

6. Kintz P., Cirimele V., Ludes B., Discrimination of the nature of doping with 19-norsteroids through hair analysis Clin. Chem. $2000 ; 46$ : 2020-2.

7. Gaillard Y., Vaysette F., Pépin G., Compared interest between hair analysis and urinalysis in doping controls. Results for amphetamines, corticosteroids and anabolic steroids in racing cyclists. Forensic Sci. Int. $2000 ; 107$ : 361-79.

8. Deng X., Kurosu A., Pounder D., Detection of anabolic steroids in head hair. J. Forensic Sci. 1999 ; 44 : 343-6.

9. Kintz P., Cirimele V., Dumestre-Toulet V., Ludes B., Doping control for nandrolone using hair analysis. J. Pharm. Biomed. Anal. $2001 ; 24$ : 1125-30. 